\title{
TIE2-expressing macrophages limit the therapeutic efficacy of the vascular-disrupting agent combretastatin A4 phosphate in mice
}

\author{
Abigail F. Welford,1,2 Daniela Biziato,,3,4 Seth B. Coffelt,, ${ }^{2}$ Silvia Nucera, ${ }^{3,4}$ Matthew Fisher, \\ Ferdinando Pucci,3,4 Clelia Di Serio,,4,5 Luigi Naldini,3,4 Michele De Palma,3 \\ Gillian M. Tozer, ${ }^{1}$ and Claire E. Lewis ${ }^{2}$

\begin{abstract}
${ }^{1}$ Tumour Microcirculation Group and ${ }^{2}$ Academic Unit of Inflammation and Tumour Targeting, The University of Sheffield Medical School, Beech Hill Road, Sheffield, United Kingdom. ${ }^{3}$ Angiogenesis and Tumor Targeting Unit, and HSR-TIGET, Division of Regenerative Medicine, Stem Cells and Gene Therapy, San Raffaele Scientific Institute, Milan, Italy. ${ }^{4}$ Vita-Salute San Raffaele University, Milan, Italy. ${ }^{5}$ University Centre of Statistics in the Biomedical Sciences (CUSSB), Milan, Italy.
\end{abstract}

\begin{abstract}
Vascular-disrupting agents (VDAs) such as combretastatin A4 phosphate (CA4P) selectively disrupt blood vessels in tumors and induce tumor necrosis. However, tumors rapidly repopulate after treatment with such compounds. Here, we show that CA4P-induced vessel narrowing, hypoxia, and hemorrhagic necrosis in murine mammary tumors were accompanied by elevated tumor levels of the chemokine CXCL12 and infiltration by proangiogenic TIE2-expressing macrophages (TEMs). Inhibiting TEM recruitment to CA4Ptreated tumors either by interfering pharmacologically with the CXCL12/CXCR4 axis or by genetically depleting TEMs in tumor-bearing mice markedly increased the efficacy of CA4P treatment. These data suggest that TEMs limit VDA-induced tumor injury and represent a potential target for improving the clinical efficacy of VDA-based therapies.
\end{abstract}

\section{Introduction}

Vascular-disrupting agents (VDAs) selectively target the tumor vasculature, resulting in a rapid and severe impairment of blood flow and secondary tumor cell death $(1,2)$. The lead VDA, combretastatin A4 phosphate (CA4P), is active in transplanted and spontaneous murine tumors as well as in human tumors $(1,3,4)$. However, viable blood vessels remaining at the tumor periphery provide a focus for regrowth and revascularization of the damaged tumor, representing treatment resistance and limiting the longterm efficacy of CA4P $(1,5)$.

Tumor-associated macrophages (TAMs) stimulate tumor angiogenesis (6), and high TAM numbers correlate with a poor prognosis in cancer patients (7). A highly proangiogenic TAM subset, the TIE2-expressing macrophages (TEMs), are present in a variety of mouse and human tumors (8-10) and physically interact with newly forming tumor blood vessels $(8,9)$ and express high levels of several proangiogenic factors $(11,12)$. Increased TEM infiltration occurs in mouse glioblastoma following high-dose radiation therapy and is associated with tumor revascularization and relapse (13). TEMs have also been implicated in the regrowth of subcutaneous breast and lung carcinomas after local irradiation (14). In the current study, we show that TEMs limit the therapeutic efficacy of CA4P in tumor-bearing mice.

\section{Results and Discussion}

Twenty-four hours after a single injection of CA4P, mammary tumors in MMTV-PyMT mice were significantly more hypoxic/ necrotic than those in controls $(P<0.05$; Figure $1, \mathrm{~A}$ and $\mathrm{B})$ and

Authorship note: Abigail F. Welford and Daniela Biziato are co-first authors. Michele De Palma, Gillian M. Tozer, and Claire E. Lewis are co-senior authors.

Conflict of interest: The authors have declared that no conflict of interest exists. Citation for this article: J Clin Invest. 2011;121(5):1969-1973. doi:10.1172/JCI44562. contained CD $31^{+}$blood vessels with smaller lumens (Figure 1C). This is consistent with the vascular-disrupting effects of CA4P reported in various mammary tumor models $(4,15)$.

The number of $\mathrm{F} 4 / 80^{+}$TAMs was also significantly increased in CA4P-treated tumors (Figure 1, D and E). F4/80, TIE2, and CD31 immunofluorescence staining of tumors showed that infiltration of CD31-F4/80+TIE2+ TEMs significantly increased 24 hours after CA4P injection (Figure 1, D and E), while the number of F4/80+TIE2- TAMs was not significantly altered (data not shown). These data were confirmed by flow cytometric analysis of dispersed tumors (Supplemental Figure 1; supplemental material available online with this article; doi:10.1172/JCI44562DS1). Virtually all $\mathrm{F} 4 / 80^{+} \mathrm{TIE} 2^{+}$TEMs expressed the proangiogenic enzyme MMP9 in both untreated and treated tumors, whereas far fewer TIE2-F4/80 ${ }^{+}$TAMs were MMP9 ${ }^{+}$(Supplemental Figure 2, A and B). CA4P treatment also significantly increased tumor infiltration by MMP9-expressing TEMs in N202 mammary tumors grown subcutaneously in syngeneic mice (Supplemental Figure 2, C and D).

Tumor expression of CXCL12 (SDF1) by both tumor and stromal cells (identified on the basis of cell morphology) increased 24 hours after CA4P treatment (Figure 2A and Supplemental Figure 3) and was consistent with the hypoxic upregulation of CXCL12 (16) and raised plasma levels of CXCL12 seen in CA4P-treated patients (17). Ninety percent of tumor TEMs and 60\% of TIE2- TAMs expressed the CXCL12 receptor, CXCR4, in MMTV-PyMT tumors (Figure 2B). CXCR4 expression per cell was significantly higher in TEMs than in TIE2- TAMs and was augmented further by CA4P treatment (Figure 2C). We then used the CXCR4 inhibitor, AMD-3100, to investigate the role of the CXCL12/CXCR4 axis in TEM recruitment to tumors after CA4P. This significantly reduced TEM but not TIE2- TAM recruitment to CA4P-treated N202 tumors (Figure 2D). Of note, AMD-3100 plus CA4P treatment elicited a significant increase in tumor necrosis compared with CA4P treatment alone (Figure 2D). 

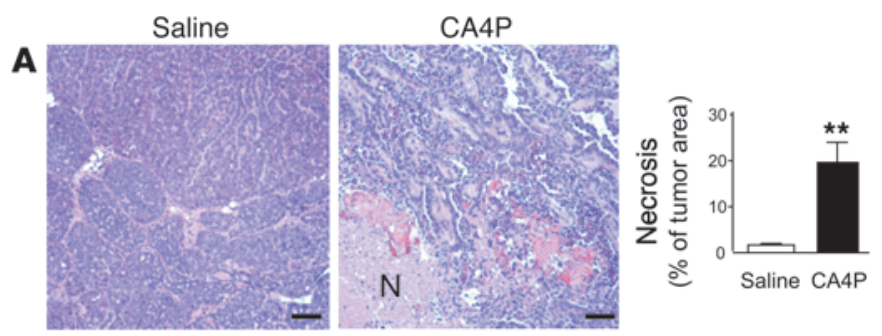

B
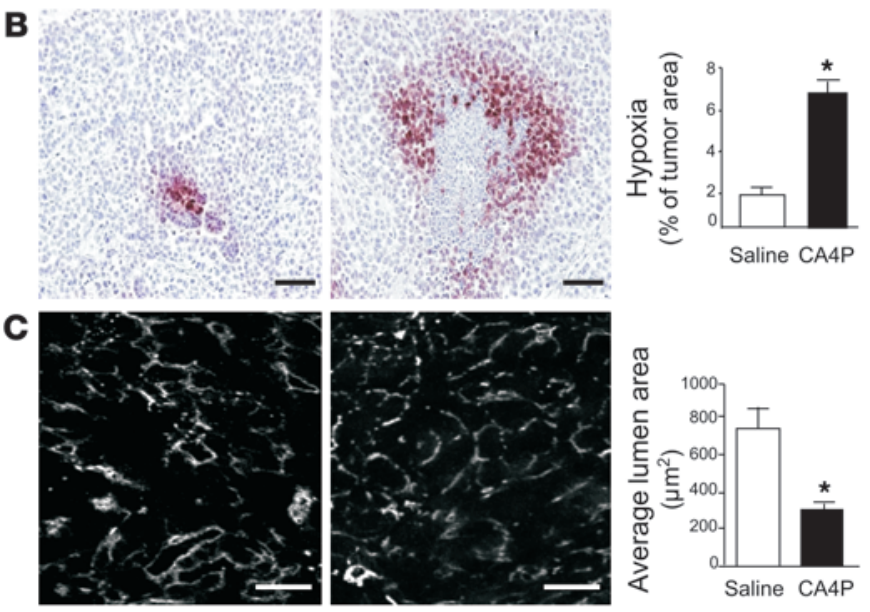

D
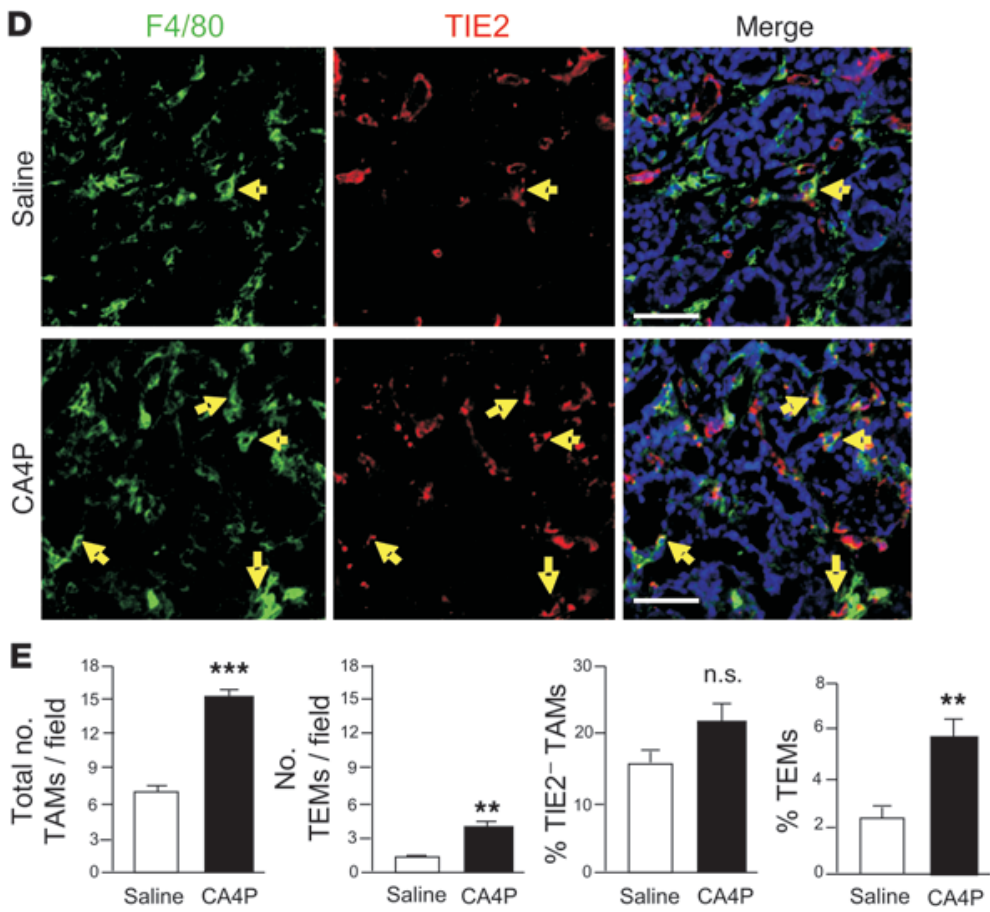

In order to study the effects of combined CXCR4 blockade and CA4P treatment on tumor responses to CA4P, we used the subcutaneous N202 mammary tumor model, which allows more reliable tumor measurements and monitoring of tumor volume than the MMTV-PyMT model. AMD-3100 alone had no effect on N202 tumor growth (Figure 2E), consistent with previous studies in other tumor models (14). The combined drug treatment (AMD-3100 and CA4P) induced a significant inhibition of N202 tumor growth compared with CA4P alone (Figure 2E).

\section{Figure 1}

CA4P induced vascular damage, increased tumor hypoxia, and increased TEM numbers in MMTV-PyMT mammary tumors. (A) H\&E staining shows a marked increase in tumor necrosis (N) 24 hours after $50 \mathrm{mg} / \mathrm{kg}$ CA4P. (B) Staining with anti-PIMO shows increased hypoxia (red). (C) Tumor sections stained with anti-CD31 antibodies show reduced lumen of blood vessels (white) 24 hours after $50 \mathrm{mg} / \mathrm{kg}$ CA4P. (D) Tumors stained with DAPI (to show cell nuclei; blue), anti-F4/80 (green), and anti-TIE2 (red). Yellow arrows denote F4/80+TIE2+ TEMs; F4/80TIE2+ structures represent blood vessels. (E) Number of all F4/80+ cells/field and F4/80+TIE2+ TEMs/field (by immunofluorescent staining; see $\mathbf{D}$ above) and proportion (\%) of $\mathrm{F} 4 / 80^{+} \mathrm{TIE}^{-}$and $\mathrm{F} 4 / 80^{+} \mathrm{TIE}^{+}$cells (TEMs) in viable cells obtained from dispersed tumors (by flow cytometric analysis; see Supplemental Figure 1). Scale bars: $50 \mu \mathrm{m}$. ${ }^{\star} P<0.05,{ }^{* *} P<0.01,{ }^{* * *} P<0.001$, compared with relevant control group. NS, no statistical difference $(P>0.05)$.

These results are consistent with the necrosis data obtained in MMTV-PyMT tumors (Figure 2D).

Because CXCR4 is not only expressed by TEMs in tumors, we also used a conditional suicide genebased strategy for specific TEM elimination (8). This involved transplanting BM cells from Tie2-HSV-thymidine kinase transgenic mice into syngeneic, wildtype mice. Six weeks after BM transplant, ganciclovir (GCV) was used to specifically eliminate TEMs in mice carrying established N202 tumors. CA4P induced an increase in necrosis (Figure $3 \mathrm{C}$ ) and vessel narrowing (Supplemental Figure 4A) in the N202 mammary tumors of transplanted mice. Coadministration of GCV to deplete TEMs resulted in significant TEM depletion in CA4P-injected tumors (Figure 3, A and B) accompanied by a striking increase in tumor necrosis in CA4P-treated tumors compared with non-TEMdepleted CA4P-treated tumors (Figure 3C). Tumor necrosis was most marked at 24 hours after CA4P in the TEM-depleted mice, indicating that TEMs are protective at early stages after treatment (Figure $3 \mathrm{C})$. Interestingly, the number of tumor-infiltrating $\mathrm{F} 4 / 80^{-} \mathrm{Gr} 1^{+}$neutrophils increased significantly at 24 hours after CA4P treatment and increased further in TEM-depleted mice that received CA4P (Supplemental Figure 4B). However, very few F4/80- Gr1 $1^{+}$neutrophils were seen at 72 hours in any of the treatment groups (Supplemental Figure 4B). Contrary to CXCR4 blockade (Figure 2D), GCV completely abolished TEM recruitment to tumors (Figure $3 \mathrm{~B}$ ) and - in agreement with previous findings (8) - markedly inhibited tumor growth (Supplemental Figure 5). It was therefore not possible to assess the additive effects of CA4P treatment and TEM elimination on tumor growth in this model.

Taken together, our results show that TEMs are rapidly recruited into CA4P-treated mammary tumors, where they have a protective effect against vascular damage, necrosis induction, and growth retardation. At present, it is not possible to distinguish whether TEMs are acting to protect against the damaging cascade of events that lead to CA4P-induced tumor necrosis or whether they are promoting rapid revascularization and tumor 
A

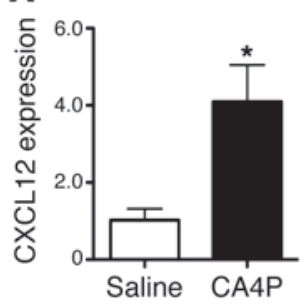

B TAMs $(60 \%)$

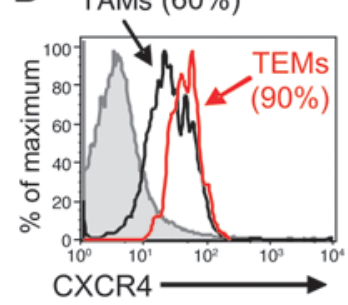

C

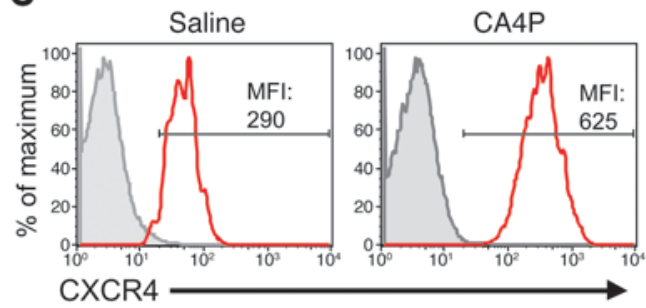

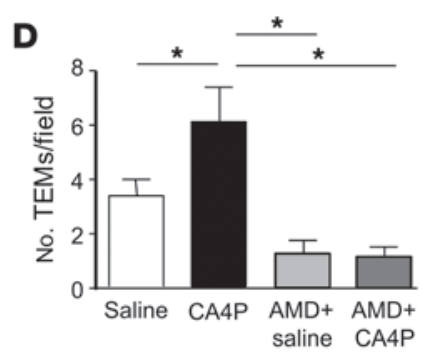
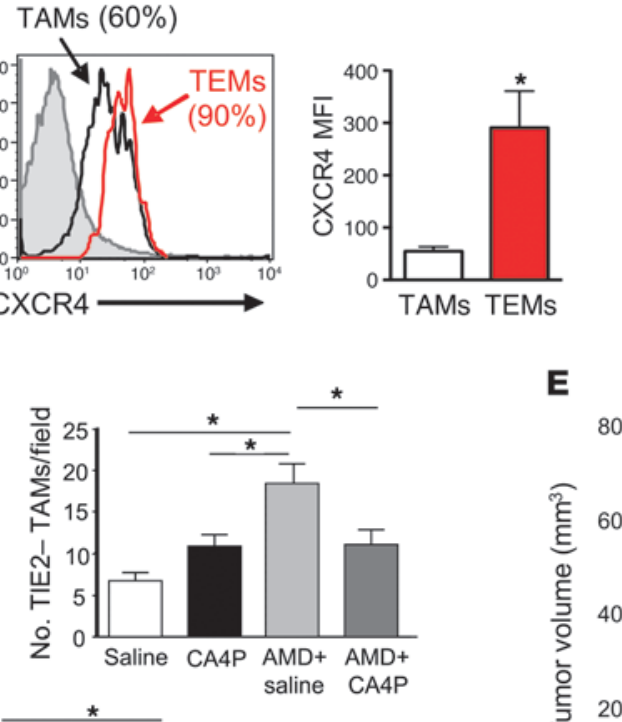

E
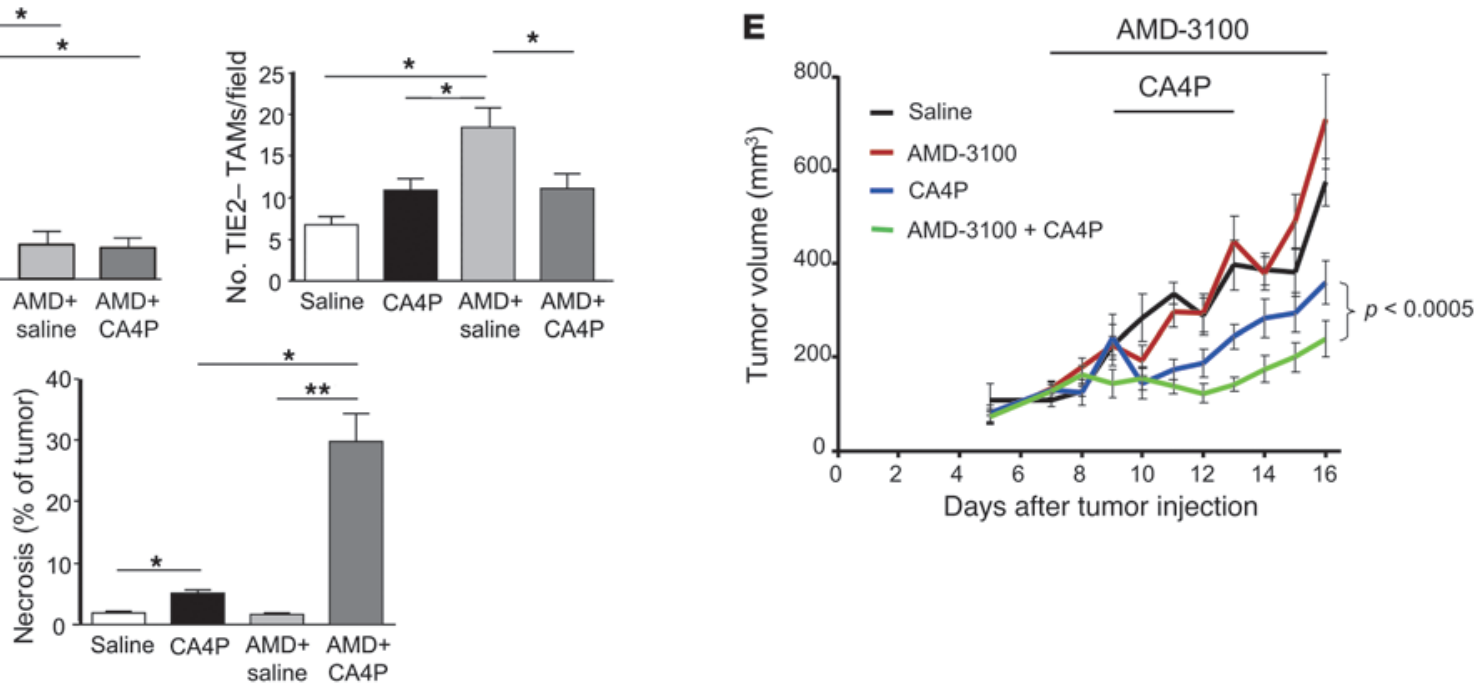

Days after tumor injection

\section{Figure 2}

Inhibition of tumor recruitment of CXCR4+ TEMs increases the therapeutic efficacy of CA4P. (A) CXCL12 expression, determined by immunofluorescence staining (see Supplemental Figure 3), was increased in MMTV-PyMT tumors 24 hours after injection with $50 \mathrm{mg} / \mathrm{kg}$ CA4P. (B and C) Flow cytometric analysis of dispersed MMTV-PyMT tumors shows that (B) the majority of F4/80+TIE2+ TEMs express CXCR4 and the MFI for CXCR4 is higher for TEMs than TIE2- TAMs and (C) CXCR4 expression/TEM is upregulated after CA4P treatment. (D) The CXCR4 inhibitor, AMD-3100, inhibits recruitment of TEMs, but not TIE2- TAMs, to MMTV-PyMT tumors and increases CA4P-induced tumor necrosis. $n>5$ mice per group. ${ }^{*} P<0.05$; ${ }^{* \star} P<0.01$. (E) Twice daily i.p. injections of $5 \mathrm{mg} / \mathrm{kg} \mathrm{AMD}-3100$ (for the period indicated) enhanced the effects of 3 daily i.p. injections of $50 \mathrm{mg} / \mathrm{kg}$ CA4P on the growth of N202 tumors. Statistical analysis in E employed the nonlinear mixed effects model described in the Supplemental Methods.

repopulation of necrotic regions. Considering the proangiogenic roles of TEMs in tumors $(8,9,12,18)$ and our finding that TEMs avidly express MMP9, we speculate that a reparative role is highly likely. Either way, our data indicate that targeting these cells is a promising approach to improving the efficacy of a VDA like CA4P.

Endothelial progenitor cells (EPCs) were previously shown to play an important role in tumor recovery following VDA treatment $(17,19)$. Shaked et al. (19) depleted EPCs using DC101, a mouse monoclonal antibody to VEGFR-2. When administered to tumor-bearing mice along with a CA4P derivative, OXi-4503 (CA1P), DC101 decreased circulating EPC numbers and tumor blood flow and increased tumor necrosis, compared with OXi4503 alone. However, DC101 would also have blocked VEGFR-2 signaling in the viable tumor blood vessels and therefore had direct effects on tumor angiogenesis that were independent of EPCs (18). Because BM-derived EPCs are very rare cells in mouse blood and tumors $(8,9,14,20-22)$, it is likely that other BMderived cell types, such as monocyte-lineage cells, are primarily involved in regulating tumor responses following VDA treatment. Although our findings do not eliminate a potential contri- bution of EPCs to tumor revascularization/recovery after CA4P, they indicate that TEMs play a crucial role in this process.

Interestingly, TEM depletion stimulated an increase in neutrophil infiltration into tumors - even in the absence of subsequent treatment with CA4P, suggesting that TEMs may inhibit tumor infiltration by $\mathrm{Gr} 1^{+} \mathrm{F} 4 / 80^{-}$neutrophils (23). CA4P treatment alone also increased neutrophil infiltration, possibly in response to tumor necrosis, and TEM depletion, in combination with CA4P, increased it even further. It is noteworthy that tumor $\mathrm{Gr}^{+}$neutrophils had significantly dropped by 72 hours, illustrating that any participation in CA4P-induced changes in tumors was time dependent. Clearly, the enhanced recruitment of neutrophils at 24 hours after treatment failed to protect tumors. Rather, neutrophils may have contributed to the increased level of necrosis present by exhibiting a cytotoxic phenotype (24). Interestingly, levels of neutrophil-derived myeloperoxidase (MPO), indicative of such a phenotype, are associated with CA4P-induced vascular injury in tumors (25).

In summary, our data show that TEMs are rapidly recruited to CA4P-treated mammary tumors and protect against the vascular damaging effects of this archetypal VDA. This suggests that targeting TEMs could increase the long-term efficacy of CA4P. 

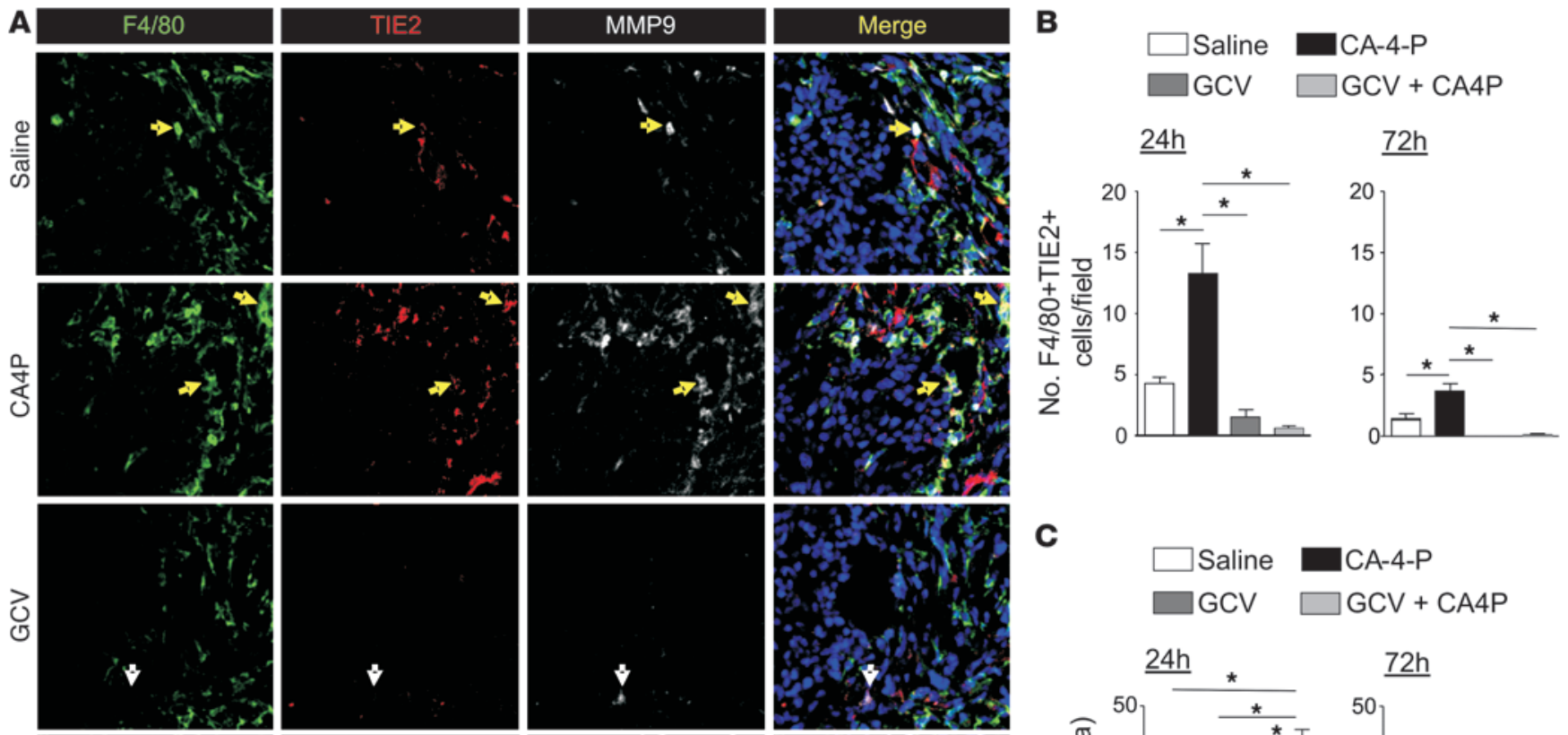

C
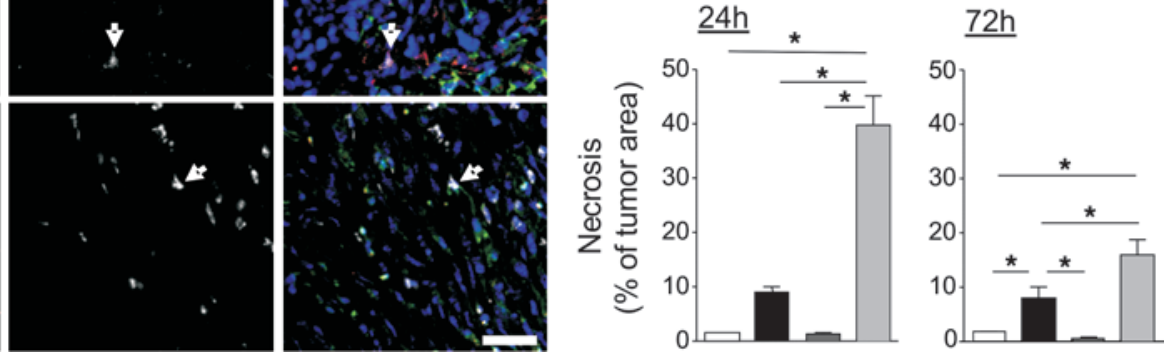

Figure 3

Conditional depletion of TEMs increases the efficacy of CA4P treatment. (A) N202 tumors excised 24 hours after the last of 3 daily i.p. injections of CA4P or saline in the presence or absence of GCV to deplete TEMs were stained with DAPI (blue), anti-F4/80 (green), anti-TIE2 (red), and anti-MMP9 (white). TEMs are highlighted with yellow arrows; non-TAMs that express MMP9 are shown with white arrows. Scale bar: 50 um. (B and C) Changes in TEM numbers and tumor necrotic area 24 hours and 72 hours after last CA4P injection. $n=6$ mice per group. ${ }^{*} P<0.05$.

\section{Methods}

See Supplemental Methods for details of methods not outlined below. All animal studies were approved by the University of Sheffield Ethics Committee/UK Home Office regulations and the Animal Care and Use Committee of the Fondazione San Raffaele del Monte Tabor, Milan, Italy.

CA4P studies. Female FVB/MMTV-PyMT mice (10-12 weeks old) were given a single i.p. injection of $50 \mathrm{mg} / \mathrm{kg}$ CA4P or saline and culled 24 hours later. Mice were also injected i.v. with $60 \mathrm{mg} / \mathrm{kg}$ pimonidazole hydrochloride (PIMO; a nitroimidazole compound used for detecting hypoxia in tumor sections) 1 hour prior to culling. Excised tumors were bisected and half frozen in OCT compound and half fixed in 10\% formalin (and then processed into paraffin wax). Some tumors were excised and enzymatically digested for FACS analysis. Mice bearing N202 mammary tumors were administered $50 \mathrm{mg} / \mathrm{kg}$ CA4P for 3 consecutive days and tumors excised 24 or 72 hours after the final dose.

AMD-3100/CA4P studies: MMTV-PyMT tumor model. MMTV-PyMT mice were injected i.p. twice daily with $5 \mathrm{mg} / \mathrm{kg}$ AMD-3100 (Sigma-Aldrich), a specific CXCR4 inhibitor. On the third day, mice were given a single i.p. injection of $50 \mathrm{mg} / \mathrm{kg}$ CA4P or saline (PBS) 2 hours after the first dose of AMD-3100. 24 hours after the last CA4P injection, mice received 1 further injection of AMD-3100 and were culled 2 hours later.

AMD-3100/CA4P studies: N202 tumor model. N202-bearing mice were injected s.c. at day 0 and $5 \mathrm{mg} / \mathrm{kg}$ AMD-3100 or saline administered twice daily i.p. starting at day 7 (when tumors reached approximately $150 \mathrm{~mm}^{3}$ ) and ending on day 16 when mice were killed and tumors removed. Daily i.p. injections of CA4P $(50 \mathrm{mg} / \mathrm{kg})$ or saline started at day 9 and ended on day 13. Tumor size was determined using callipers.

TEM conditional depletion studies. Six-week-old Tie2-HSV-tk transgenic mice (8) were sacrificed with $\mathrm{CO}_{2}$ and their $\mathrm{BM}$ harvested by flushing the femurs and the tibias. BM cells $\left(10^{7} /\right.$ mouse) were infused into the tail veins of 40 lethally irradiated (975 cGy) 6-week-old FVB mice. Six weeks after transplant, we confirmed hematopoietic reconstitution of recipient mice by quantitative PCR (qPCR) analysis of vector sequences in blood leukocytes, as described $(8,9)$. N202 mammary tumor cells $\left(5 \times 10^{6}\right)$ were then injected s.c. into the flanks of BM-transplanted mice (day 0 ). Thirteen days (day 13) after N202 tumor challenge (average tumor volume: $700 \mathrm{~mm}^{3}$ ), transplanted mice were treated with GCV $(100 \mathrm{mg} / \mathrm{kg})$ daily for 11 days or remained untreated. Tumor size was determined using callipers. Nineteen days (day 19) after N202 tumor cell inoculation, GCV-injected and nonGCV-injected mice were randomly allocated to receive CA4P $(50 \mathrm{mg} / \mathrm{kg})$ or saline vehicle for 3 consecutive days; they were culled 24 or 72 hours later and tumors excised and processed as above.

Statistics. In most cases, multiple comparison of groups was performed by ANOVA followed by the Tukey-Kramer honest significance difference test (GraphPad Software Inc.). See figure legends for any alternative statistical tests used. The non-linear mixed effects (NLME) model used for the tumor growth curves in Figure 2E was implemented using the R statistical software, version 2.9.1 (The R Project for Statistical Computing; http://www. r-project.org/) (see Supplemental Methods). All data represent mean values $\pm \mathrm{SEM} ; P$ values of less than 0.05 were considered to be significant. 


\section{Acknowledgments}

The authors thank the following funding bodies for their support: Cancer Research UK (to A.F. Welford, C.E. Lewis, and G.M. Tozer), European Research Council (to M. De Palma), and Associazione Italiana per la Ricerca sul Cancro (to L. Naldini). They thank Zena Werb at UCSF for the breeding pairs of MMTV-PyMT mice and anti-MMP9 antibody, Carlos Reyes-Aldasoro for help with image analysis, and Bob Pettit at Arizona State University for the supply of CA4P.

Received for publication July 29, 2010, and accepted in revised form January 26, 2011.

1. Dark GG, Hill SA, Prise VE, Tozer GM, Pettit GR, Chaplin DJ. Combretastatin A-4, an agent that displays potent and selective toxicity toward tumour vasculature. Cancer Res. 1997;57(10):1829-1834.

2. Tozer GM, et al. Combretastatin A-4 phosphate as a tumor vascular-targeting agent: early effects in tumors and normal tissues. Cancer Res. 1999; 59(7):1626-1634

3. Hill SA, Chaplin DJ, Lewis G, Tozer GM. Schedule dependence of combretastatin A4 phosphate in transplanted and spontaneous tumour models. Int J Cancer. 2002;102(1):70-74.

4. Galbraith SM, Maxwell RJ, Lodge MA, Tozer GM, Wilson J, Taylor NJ. Combretastatin A4 phosphate has tumour antivascular activity in rat and man as demonstrated by dynamic magnetic resonance imaging. J Clin Oncol. 2003;21(15):2831-2842.

5. Tozer GM, et al. Mechanisms associated with tumor vascular shut-down induced by combretastatin A-4 phosphate: intravital microscopy and measurement of vascular permeability. Cancer Res. 2001;61(17):6413-6422.

6. Lin EY, et al. Macrophages regulate the angiogenic switch in a mouse model of breast cancer. Cancer Res. 2006;66(23):11238-11246.

7. Bingle L, Brown NJ, Lewis CE. The role of tumourassociated macrophages in tumour progression: implications for new anticancer therapies. J Pathol. 2002;196(3):254-265.

8. De Palma M, Venneri MA, Galli R. Tie2 identifies a hematopoietic lineage of proangiogenic monocytes required for tumour vessel formation and a mesenchymal population of pericyte progenitors. Cancer Cell. 2005;8(3):211-226.

Address correspondence to: Claire E. Lewis, Floor E, The University of Sheffield Medical School, Beech Hill Road, Sheffield, S10 2RX, United Kingdom. Phone: 44.114.271.2903; Fax: 44.114.271.2903; E-mail: Claire.lewis@sheffield.ac.uk. Or to: Gill Tozer, Floor K, The University of Sheffield Medical School, Beech Hill Road, Sheffield, S10 2RX, United Kingdom. Phone: 44.114.271.2423; Fax: 44.114.271.3314; E-mail: G.Tozer@sheffield.ac.uk. Or to: Michele De Palma, Angiogenesis and Tumor Targeting Unit, Division of Regenerative Medicine, Stem Cells and Gene Therapy, and HSR-TIGET, San Raffaele Scientific Institute, Olgettina, 58, 20132-Milan, Italy. Phone: 39.02.2643.5680; Fax: 39.02.2643.4621; E-mail: depalma.michele@hsr.it.

9. De Palma M, Venneri MA, Roca C, Naldini L. Targeting exogenous genes to tumour angiogenesis by transplantation of genetically modified hematopoietic stem cells. Nat Med. 2003;9(6):789-795.

10. Venneri MA, et al. Identification of proangiogenic TIE2-expressing monocytes (TEMs) in human peripheral blood and cancer. Blood. 2007;109(12):5276-5285.

11. Coffelt SB, et al. Angiopoietin-2 Regulates Gene Expression in TIE2-Expressing Monocytes and Augments Their Inherent Proangiogenic Functions. Cancer Res. 2010;70(13):5270-5280.

12. Pucci $F$, et al. A distinguishing gene signature shared by tumor-infiltrating TIE2-expressing monocytes, blood "resident" monocytes, and embryonic macrophages suggests common functions and developmental relationships. Blood. 2009;114(4):901-914.

13. Kioi M, Vogel H, Schultz G, Hoffman RM, Harsh GR, Brown JM. Inhibition of vasculogenesis, but not angiogenesis, prevents the recurrence of glioblastoma after irradiation in mice. J Clin Invest. 2010;120(3):694-705.

14. Kozin SV, Kamoun WS, Huang Y, Dawson MR, Jain RK, Duda DG. Recruitment of myeloid but not endothelial precursor cells facilitates tumor regrowth after local irradiation. Cancer Res. 2010; 70(14):5679-5685.

15. Murata R, Overgard J, Horsman MR. Comparative effects of combretastatin a- 4 disodium phosphate and 5,6-dimethylxanethenone-4-acetic acid on blood perfusion in a murine tumour and normal tissues Int J Radiat Biol. 2001;77(2):195-204.

16. Ceradini DJ, et al. Progenitor cell trafficking is regulated by hypoxic gradients through $\mathrm{HIF}-1$ induc- tion of SDF-1. Nat Med. 2004;10(8):858-864.

17. Shaked Y, et al. Contribution of granulocyte colony-stimulating factor to the acute mobilization of endothelial precursor cells by vascular disrupting agents. Cancer Res. 2009;69(19):7524-7528.

18. Fantin A, et al. Tissue macrophages act as cellular chaperones for vascular anastomosis downstream of VEGF-mediated endothelial tip cell induction. Blood. 2010;116(5):829-840.

19. Shaked Y, et al. Therapy-induced acute recruitment of circulating endothelial progenitor cells to tumors. Science. 2006;313(5794):1785-1787.

20. Ahn GO, Brown JM. Matrix metalloproteinase-9 is required for tumor vasculogenesis but not for angiogenesis: role of bone marrow-derived myelomonocytic cells. Cancer Cell. 2008;13(3):193-205.

21. Shojaei $F$, et al. Tumor refractoriness to anti-VEGF treatment is mediated by $\mathrm{CD} 11 \mathrm{~b}+\mathrm{Gr} 1+$ myeloid cells. Nat Biotechnol. 2007;25(8):911-920.

22. Dudley AC, et al. Bone marrow is a reservoir for pro-angiogenic myelomonocytic cells but not endothelial cells in spontaneous tumors. Blood. 2010;116(17):3367-3371.

23. Pahler JC, et al. Plasticity in tumor-promoting inflammation: impairment of macrophage recruitment evokes a compensatory neutrophil response. Neoplasia. 2008;10(4):329-340.

24. Fridlender $Z G$, et al. Polarization of tumor-associated neutrophil phenotype by TGF-beta: "N1" versus “N2” TAN. Cancer Cell. 2009;16(3):183-194.

25. Parkins CS, Holder AJ, Hill SA, Chaplin DJ, Tozer GM. Determinants of anti-vascular action by combretastatin A-4-phosphate:role of nitric oxide. $\mathrm{BrJ}$ Cancer. 2000;83(6):811-816. 